\title{
Low and High-Delay Source-Channel Coding with Bandwidth Expansion and Correlated Interference
}

\author{
Ahmad Abou Saleh, Wai-Yip Chan, and Fady Alajaji \\ Queen's University, Kingston, ON, K7L 3N6 \\ Email: ahmad.abou.saleh@queensu.ca,chan@queensu.ca, fady@mast.queensu.ca
}

\begin{abstract}
We consider the problem of sending a Gaussian source over an additive white Gaussian noise channel with Gaussian correlated interference known to the transmitter. We study both low-delay and asymptotically high-delay (in the sense of infinite source and coding block lengths) joint source-channel coding schemes based on purely analog and hybrid-digital analog (HDA) schemes with bandwidth expansion, respectively. The achievable (square error) distortion region of these schemes under matched and mismatched noise power is analyzed. The low-delay scheme uses a non-parametric analog mapping that is designed using a joint optimization of the encoder and the decoder. Numerical results show that the non-parametric approach adapts better to the interference than the classical linear scheme. For the high-delay regime, we establish a lower bound on the system's distortion and propose a layered HDA scheme based on Wyner-Ziv and HDA Costa coding. The proposed HDA scheme is shown to perform close to the derived bound and to be resilient under noise mismatch.
\end{abstract}

\section{INTRODUCTION}

The traditional approach for analog source transmission is to use separate source and channel coders. This separation is optimal given unlimited delay and complexity in the coders. On the other hand, joint source-channel coding (JSCC) can lead to a better performance when delay and complexity are constrained and in the case of mismatched noise level between transmitter and receiver. It is well known that for the case of a Gaussian source sent over a Gaussian channel with matched bandwidth, uncoded (linear) transmission is optimal. However, when there is a bandwidth mismatch between source and channel or in the presence of interference known only at the transmitter, this result does not hold anymore. A tandem digital Costa coding scheme which comprises an optimal quantizer followed by a Costa's dirty paper coding [1] is optimal in the absence of correlation between the source and the interference. This scheme, however, suffers from the threshold effect and the levelling-off effect in the high and low noise power regimes, respectively [2]. A family of hybrid digital-analog (HDA) schemes are introduced in [3], [4] that overcome the threshold and the levelling-off effect. In [5], an HDA Costa coding scheme is proposed for the case of matched bandwidth between source and channel over the additive white Gaussian noise (AWGN) channel with uncorrelated interference. In [6], the authors study the HDA schemes proposed in [5] for broadcasting correlated sources. In [7], the authors adapt the schemes of [5] for bandwidth reduction. Recently, the authors in [8] consider the transmission of Gaussian sources over

This work was supported in part by NSERC of Canada.
AWGN channels with correlated interference, and propose a scheme based on HDA Costa coding [5] under matchedbandwidth. All these schemes require high delay and complexity due to the use of long block codes.

With the increasing popularity of wireless sensor networks (WSNs), reliable transmission with delay and complexity constraints has become an important practical objective. A sensor node, often conceived as having limited lifetime and processing power, communicates its sensed information to a fusion centre over a noisy wireless channel. Low-delay as well as analog coding comport with low power implementation. In [9]-[14], the authors present low-delay JSCC schemes based on purely analog mappings over noisy channels with no interference. In [15], the authors consider lossless coding of a uniformly distributed finite-alphabet source that is uncorrelated with the interference and propose a symbol-bysymbol modulation technique that minimizes the symbol error probability.

In this work, we study the reliable transmission of a memoryless Gaussian source over the AWGN channel with correlated interference known non-causally [1] at the transmitter. More precisely, we consider both low-delay and high-delay schemes with bandwidth expansion. We analyze the achievable distortion region under matched and mismatched noise levels. One application of low-delay JSCC with correlated interference can be found in WSNs where two sensors interfere with each other. One sensor transmits directly its sensed signal; the other, however, is able to detect its neighbour sensor transmission and treat it as a correlated interference. The rest

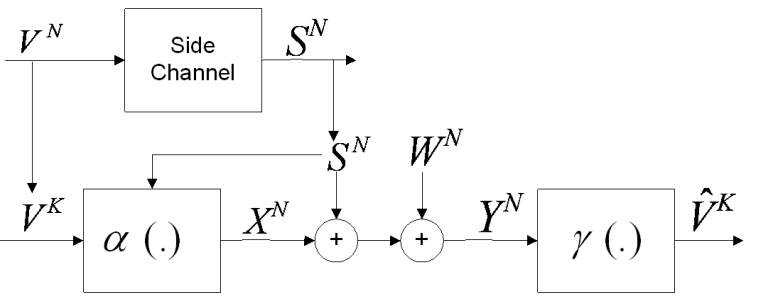

Fig. 1. A $K: N$ system structure (with $K<N$ ) over the AWGN channel with interference known at the transmitter. The interference $S^{N}$ is assumed to be the output of a noisy side channel with input $V^{N} . V^{K}$ represents the first $K$ samples of $V^{N}$. Note that the first $K$ pairs $(V, S)$ are correlated.

of the paper is organized as follows. Section II presents the problem formulation. Section III describes the lower bound on system's distortion and some reference schemes. In Section IV, a low-delay scheme is considered. Section V presents the achievable distortion region for a high-delay HDA scheme under both matched and mismatched noise levels. Finally, 
conclusions are drawn in Section VI.

\section{PRoblem Formulation}

We consider the transmission of a Gaussian source $V^{K}=$ $\left(V_{1}, \ldots, V_{K}\right)^{T} \in \mathbb{R}^{K}$ over an AWGN channel in the presence of Gaussian interference $S^{N}=\left(S_{1}, \ldots, S_{N}\right)^{T} \in \mathbb{R}^{N}$ known at the transmitter, where $(.)^{T}$ denotes the transpose operator and $N>K$. As shown in Fig. 1, the source vector $V^{K}$, which is composed of independent and identically distributed (i.i.d.) samples, is transformed into an $N$ dimensional channel input $X^{N} \in \mathbb{R}^{N}$ using a nonlinear mapping function, in general, $\alpha(\cdot): \mathbb{R}^{K} \times \mathbb{R}^{N} \rightarrow \mathbb{R}^{N}$. The received symbol is

$$
Y^{N}=X^{N}+S^{N}+W^{N}
$$

where addition is component-wise, $X^{N}=\alpha\left(V^{K}, S^{N}\right), S^{N}$ is an i.i.d. Gaussian interference $\left(S_{i} \sim \mathcal{N}\left(0, \sigma_{S}^{2}\right), i=1, \ldots, N\right)$ and considered to be the output of a side channel with input $V^{N}$ as shown in Fig. 1, and each sample in the additive noise $W^{N}$ is drawn from a Gaussian distribution $\left(W \sim \mathcal{N}\left(0, \sigma_{W}^{2}\right)\right)$ independently from both $S$ and $V$. Unlike the typical dirty paper problem, $V^{K}$ and $S^{N}$ are correlated and have a joint probability density function denoted by $p\left(V^{K}, S^{N}\right)$. In this work, we assume that $V_{i}$ and $S_{i}, i=1, \ldots, K$, are correlated with the following covariance matrix

$$
\Sigma_{V S}=\left[\begin{array}{cc}
\sigma_{V}^{2} & \rho \sigma_{V} \sigma_{S} \\
\rho \sigma_{V} \sigma_{S} & \sigma_{S}^{2}
\end{array}\right]
$$

where $\sigma_{V}^{2}, \sigma_{S}^{2}$ are, respectively, the variance of the source and the interference, and $\rho$ is the correlation coefficient. The system operates under an average power constraint $P$

$$
\mathbb{E}\left[\left\|\alpha\left(V^{K}, S^{N}\right)\right\|^{2}\right] / N \leq P
$$

where $\mathbb{E}[(\cdot)]$ denotes the expectation operator. The reconstructed signal is given by $\hat{V}^{K}=\gamma\left(Y^{N}\right)$, where the decoder is a mapping from $\mathbb{R}^{N} \rightarrow \mathbb{R}^{K}$. We aim to find a source mapping $\alpha$ and receiver $\gamma$ that minimize the mean square error (MSE) distortion $D=\mathbb{E}\left[\left\|V^{K}-\hat{V}^{K}\right\|^{2}\right] / K$ under the average power constraint in (3). The system rate is given by $r=\frac{N}{K}$ channel use/source symbol. In this work, we are interested in analyzing the achievable distortion for bandwidth expansion $(r>1)$ under both low-delay (where both $K$ and $N$ take on small values above unity) and high-delay regimes (where both $N$ and $K$ tend to infinity such that $\frac{N}{K}=r$ is constant).

- Low-delay regime: This regime uses a non-parametric source-channel analog mapping ${ }^{1}$ based on a joint optimization between the encoder and the decoder side through an iterative process.

- High-delay regime: A layered coding scheme based on HDA Costa [5], [8] and Wyner-Ziv coding is proposed for bandwidth expansion. The achievable distortion region is analyzed under matched and mismatched noise levels. Moreover, a lower bound on the system's distortion is derived.

\section{LOWER BOUND AND REFERENCE SySTEMS}

\section{A. Lower Bound}

In [8], [16], several lower bounds on the system distortion for the case of matched-bandwidth are derived by assuming

\footnotetext{
${ }^{1}$ For efficient implementation purposes, our mappings are not purely analog; rather, they are discretized close-to-analog maps.
}

full/partial knowledge of the interference at the decoder side. Since $S^{K}$ and $V^{K}$ are correlated and Gaussian, we have $S^{K}=$ $S_{I}^{K}+S_{D}^{K}$, with $S_{D}^{K}=\frac{\rho \sigma_{S}}{\sigma_{V}} V^{K}$ and $S_{I} \sim \mathcal{N}\left(0,\left(1-\rho^{2}\right) \sigma_{S}^{2}\right)$. To derive a distortion lower bound, we assume knowledge of $\tilde{S}^{K}$ and $S^{N-K}$ (the last $N-K$ samples in $S^{N}$ ) at the decoder side, where $\tilde{S}^{K}=\beta_{1} S_{I}^{K}+\beta_{2} S_{D}^{K}$. The knowledge of the linear combination $\tilde{S}$ is motivated by [16]. This lower bound can be written as follows

$$
D \geq \frac{\operatorname{Var}(V \mid \tilde{S})\left(\sigma_{W}^{2}\right)^{r}}{(\operatorname{MSE}(Y ; \tilde{S}))\left(P+\sigma_{W}^{2}\right)^{r-1}}
$$

where $\operatorname{Var}(V \mid \tilde{S})=\sigma_{V}^{2}\left(1-\frac{\beta_{2}^{2} \rho^{2}}{\beta_{1}^{2}\left(1-\rho^{2}\right)+\beta_{2}^{2} \rho^{2}}\right)$ and $\operatorname{MSE}(Y ; \tilde{S})$ is the distortion from estimating $Y$ based on $\tilde{S}$ using a linear minimum MSE estimator. This distortion is a function of $\beta_{1}, \beta_{2}, \mathbb{E}\left[X S_{I}\right]$ and $\mathbb{E}\left[X S_{D}\right]$. By Cauchy-Schwartz, we have $\left|\mathbb{E}\left[X S_{I}\right]\right| \leq \sqrt{\mathbb{E}\left[X^{2}\right] \mathbb{E}\left[S_{I}^{2}\right]}$ and $\left|\mathbb{E}\left[X S_{D}\right]\right| \leq \sqrt{\mathbb{E}\left[X^{2}\right] \mathbb{E}\left[S_{D}^{2}\right]}$. For a given $\beta_{1}$ and $\beta_{2}$, the maximum value of $\operatorname{MSE}(Y ; \tilde{S})$ has to be used in (4). Note that we need to maximize $D$ over the parameters $\beta_{1}$ and $\beta_{2}$. This bound reduces to the ones derived in [16] for the matched case $(r=1)$.

\section{B. Linear Scheme}

In this scheme, the encoder transforms the $K$-dimensional signal $V^{K}$ into an $N$-dimensional channel input $X^{N}$ using a linear transformation according to

$$
X^{N}=\alpha\left(V^{K}, S^{N}\right)=\mathbf{A} V^{K}+\mathbf{B} S^{N}
$$

where $\mathbf{A}$ is a $N \times K$ matrix and $\mathbf{B}$ is a $N \times N$ matrix. At the receiver side, we use a linear decoder that minimizes the MSE distortion. The estimated source is $\hat{V}^{K}=F E^{-1} Y^{N}$, where $F$ is the correlation vector between $V^{K}$ and $Y^{N}$, and $E$ is the covariance matrix of $Y^{N}$. By setting $\mathbf{B}$ to be the zero matrix, the system reduces to the uncoded scheme which do not use the interference knowledge. Note that $\mathbf{A}$ and $\mathbf{B}$ are found by minimizing the MSE distortion while satisfying (3).

\section{Tandem Digital Costa Scheme}

This scheme is based on the concatenation of an optimal source code and Costa (dirty paper) [1]. The optimal source code quantizes the analog source with a rate close to the channel capacity, and the Costa coder achieves a rate equal to the case when there is no interference. Hence, from the lossy JSCC theorem, the distortion for a $K: N$ system is

$$
D_{\text {Costa }}=\sigma_{V}^{2} /\left(1+P / \sigma_{W}^{2}\right)^{r} .
$$

Note that this scheme achieves the theoretical limit for uncorrelated interference $(\rho=0)$. Moreover, its performance does not improve when the noise level decreases (levellingoff effect) or in the presence of correlation between $V$ and $S$ $(\rho \neq 0)$.

\section{LOW-DELAY MAPPINGS}

\section{A. Non-Parametric Mappings}

Using the Lagrange multiplier method, the constrained optimization problem of minimizing the MSE $\mathbb{E}\left[\left\|V^{K}-\hat{V}^{K}\right\|^{2}\right] / K$ distortion subject to (3) can be recast into an unconstrained minimization problem via the Lagrange cost function $L(\alpha, \gamma)$

$$
L(\alpha, \gamma)=\mathbb{E}\left[\left\|V^{K}-\hat{V}^{K}\right\|^{2}\right] / K+\lambda \mathbb{E}\left[\left\|\alpha\left(V^{K}, S^{N}\right)\right\|^{2}\right] / N
$$


where the Lagrange multiplier $\lambda$ is used to control the average power. However, this unconstrained minimization is still hard to solve due to the interdependencies between the optimized components, and since the encoder/decoder mappings are, in general, nonlinear functions. To overcome these challenges, we proceed in a similar way to classical design problems (e.g., channel optimized vector quantizer design [17]) by formulating the necessary conditions for optimality. This is done by finding the optimal encoder $\alpha$ given the decoder $\gamma$, and vice versa.

1) Necessary Conditions for Optimality: The problem of finding the optimal source mapping $\alpha$ (while $\gamma$ is fixed) is $\alpha^{*}=\arg \min _{\alpha}\left\{\frac{\mathbb{E}\left[\left\|V^{K}-\hat{V}^{K}\right\|^{2}\right]}{K}+\lambda \frac{\mathbb{E}\left[\left\|\alpha\left(V^{K}, S^{N}\right)\right\|^{2}\right]}{N}\right\}$.

Using Bayes' rule, the distortion $D=\frac{\mathbb{E}\left[\left\|V^{K}-\hat{V}^{K}\right\|^{2}\right]}{K}$ is

$D=\iiint p\left(v^{k}, s^{n}\right) p\left(y^{n} \mid \alpha\left(v^{k}, s^{n}\right), s^{n}\right) \frac{\left\|v^{k}-\hat{v}^{k}\right\|^{2}}{K} d v^{k} d s^{n} d y^{n}$

where $p($.$) and p(. \mid$.$) denote, respectively, a probability density$ function (pdf) and a conditional pdf. The average power is

$$
P=\frac{1}{N} \iint p\left(v^{k}, s^{n}\right)\left\|\alpha\left(v^{k}, s^{n}\right)\right\|^{2} d v^{k} d s^{n} .
$$

Since $p\left(v^{k}, s^{n}\right)$ in (9)-(10) is nonnegative, the optimal source mapping $\alpha$ can be optimized for each $v^{k}$ and $s^{n}$ according to

$$
\begin{aligned}
\alpha^{*}\left(v^{k}, s^{n}\right)= & \arg \min _{x^{n} \in \mathbb{R}^{n}}\left\{\frac{1}{K} \int p\left(y^{n} \mid x^{n}, s^{n}\right)\right. \\
& \left.\left\|v^{k}-\gamma\left(y^{n}\right)\right\|^{2} d y^{n}+\frac{\lambda}{N}\left\|x^{n}\right\|^{2}\right\} .
\end{aligned}
$$

Hence, (11) is a necessary condition to find the optimal $\alpha$.

On the receiver side, the optimal decoder in the MSE sense (assuming $\alpha$ is fixed) is given by

$$
\gamma^{*}\left(y^{N}\right)=\mathbb{E}\left[V^{K} \mid y^{n}\right]=\frac{\iint v^{k} p\left(y^{n} \mid v^{k}, s^{n}\right) p\left(v^{k}, s^{n}\right) d v^{k} d s^{n}}{\iint p\left(y^{n} \mid v^{k}, s^{n}\right) p\left(v^{k}, s^{n}\right) d v^{k} d s^{n}} .
$$

2) Design Algorithm: Based on the above necessary conditions for optimality, it is possible to optimize the transmitter mapping $\alpha$ and the receiver mapping $\gamma$ using an iterative process. This is done by fixing one part while optimizing the other. The update equations (11) and (12) yield lower distortion at each iteration step. One common problem with such iterative technique is that the final solution will depend on the initialization of the algorithm and does not guarantee convergence to the global optimum solution.The design (offline) algorithm is as follows

1) Choose some initial mapping for the encoder $\alpha$.

2) Find the optimal receiver $\gamma$ according to (12).

3) Set the iteration index $i=0$ and the cost $L^{(0)}=\infty$.

4) Set $i=i+1$.

5) Find the optimal mapping $\alpha$ according to (11).

6) Find the optimal receiver $\gamma$ according to (12).

7) Evaluate the cost function $L^{(i)}$. If the relative improvement of $L^{(i)}$ compared to $L^{(i-1)}$ is less than some positive threshold $\varepsilon$, stop iterating. Else go to step 4 .

In our simulations, we used $\varepsilon=10^{-4}$, an uncoded mapping for initializing the encoder $\alpha$, and $\lambda$ is chosen so that the average power constraint is satisfied. Note that the $\lambda$ value to use in the operational phase (i.e., when the encoder is deployed) is obtained from the design phase; that is the $\lambda$ values found in the search for a given power constraint and noise levels are tabulated for implementing (11) online.

3) Implementation Aspects: For the actual implementation of (11) and (12), some modifications are required. By the fact that it is impossible to evaluate the formulas for all $\left(V^{K}, S^{N}\right)$, we form as in [18] a pair of sets $(\mathcal{V}, \mathcal{S})$ composed of MonteCarlo samples drawn from the joint distribution of $V^{K}$ and $S^{N}$. In our simulations, we use $10^{4}$ sample pairs to define $(\mathcal{V}, \mathcal{S})$. Since the channel input and output spaces are real valued, we discretize each coordinate of these spaces using a pulse amplitude modulation (PAM) alphabet $\mathcal{X}$

$$
\mathcal{X}=\left\{-e \frac{\ell-1}{2},-e \frac{\ell-3}{2}, \ldots, e \frac{\ell-3}{2}, e \frac{\ell-1}{2}\right\}
$$

where $e$ and $\ell$ determine the resolution and the cardinality of the discrete set $\mathcal{X}$, respectively. This set provides a good approximation by taking $e$ to be small in relation to the standard deviation of the noise and by choosing a sufficiently large $\ell$ to cover the largest signal amplitude. In our simulations, we set $e=12 /(\ell-1)$, and $\ell$ to 500 for 1:2 expansion system. ${ }^{2}$

Since complexity is a major concern for the low-delay scheme, it is important to note that the encoder side can be approximated with a table-lookup, thereby avoiding having to compute a numerical integration for each $(V, S)$.

The discretized versions of (11) and (12), that are used in the implementation of the design algorithm, are

$$
\begin{aligned}
\alpha^{*}\left(v^{k}, s^{n}\right)= & \arg \min _{x^{n} \in \mathcal{X}^{n}}\left\{\sum_{y^{n} \in \mathcal{X}^{n}} \frac{1}{K} Q\left(y^{n} \mid x^{n}, s^{n}\right)\right. \\
& \left.\left\|v^{k}-\gamma\left(y^{n}\right)\right\|^{2}+\frac{\lambda}{N}\left\|x^{n}\right\|^{2}\right\} \\
\gamma^{*}\left(y^{n}\right)= & \frac{\sum_{\left(v^{k}, s^{n}\right) \in(\mathcal{V}, \mathcal{S})} v^{k} Q\left(y^{n} \mid \alpha\left(v^{k}, s^{n}\right), s^{n}\right)}{\sum_{\left(v^{k}, s^{n}\right) \in(\mathcal{V}, \mathcal{S})} Q\left(y^{n} \mid \alpha\left(v^{k}, s^{n}\right), s^{n}\right)}
\end{aligned}
$$

respectively, where $Q(\cdot)$ and $Q(\cdot \mid \cdot)$ denote a probability mass function (pmf) and a conditional pmf, respectively.

\section{B. Numerical Results}

In this section, we assume an i.i.d. zero-mean Gaussian source with unitary variance that is transmitted over an AWGN channel with Gaussian interference $S$. The interference power is $\sigma_{S}^{2}=1$ and the power constraint is $P=1$. To evaluate the performance, we show plots of the signal-to-distortion ratio $\left(\mathrm{SDR} \triangleq \mathbb{E}\left[\left\|V^{K}\right\|^{2}\right] / \mathbb{E}\left[\left\|V^{K}-\hat{V}^{K}\right\|^{2}\right]\right)$ versus $\mathrm{CSNR} \triangleq P / \sigma_{W}^{2}$. Fig 2 shows the performance of non-parametric mappings for the 1:2 bandwidth expansion system and $\rho=0.3$. We can notice that the non-parametric mappings outperform the uncoded and the linear schemes for all CSNR levels. The gain from using the non-parametric mapping is due to the fact that it has a higher degree of freedom in placing points in space. Other numerical results show that the system performance increases with the correlation between the source and the interference which is not true for the digital Costa scheme. It should be emphasized that the performance of the tandem Costa scheme is an asymptotic result in the sense of infinite

\footnotetext{
${ }^{2}$ Note that $\ell$ depends on the noise levels; for high noise levels, $\ell$ can be made smaller with no loss in performance.
} 
source and coding block lengths (but keeping $N / K=r$ constant). The gap between the digital Costa and our lowdelay schemes is therefore not surprising.

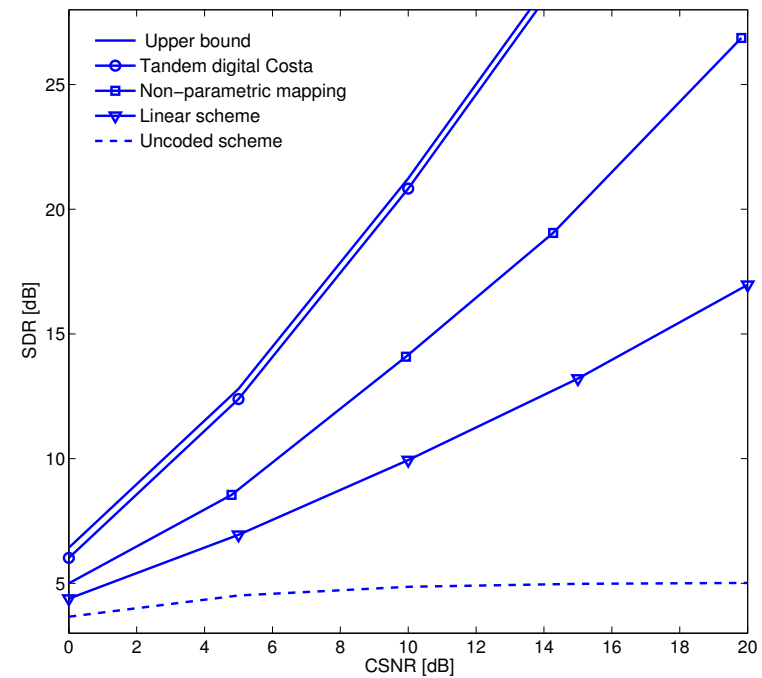

Fig. 2. Performance of the non-parametric mapping for 1:2 bandwidth expansion. Tandem Costa, uncoded, and linear schemes are also plotted. The graph is made for $\sigma_{V}^{2}=1, P=1, \rho=0.3$, and $\sigma_{S}^{2}=1$.

\section{Distortion Region fOR High-Delay Schemes}

\section{A. HDA Scheme for Bandwidth Expansion}

As shown in Fig. 3, this scheme comprises two layers, a hybrid layer (lower part) outputs $X_{1}^{K}$, and a purely digital layer (upper part) that outputs $X_{2}^{N-K}$. The transmitted sequence is obtained by multiplexing the output codeword of both layers. In the purely digital layer, the $K$ source samples $V^{K}$ are first encoded using a Wyner-Ziv encoder that produces an index $m$. Then, the index is transmitted using Costa coding that treats $S^{N-K}\left(S^{N}=\left[S^{K}, S^{N-K}\right]\right)$ as an interference. The output of this layer is denoted by $X_{2}^{N-K}$. The other (hybrid) layer consists of two sublayers as illustrated in Fig. 3. The first sublayer has an average power $P_{a}$ and outputs $X_{a}^{K}=\sqrt{a}\left(\beta_{1} V^{K}+\beta_{2} S^{K}\right)$, where $\beta_{1}, \beta_{2} \in[-11]$, and $a=P_{a} /\left(\beta_{1} \sigma_{V}^{2}+\beta_{2}^{2} \sigma_{S}^{2}+2 \beta_{1} \beta_{2} \rho \sigma_{V} \sigma_{S}\right)$ is a gain factor related to power constraint $P_{a}$. The second sublayer encodes $V^{K}$ using the HDA Costa coder [5], [8] with an average power $P_{h}=P-P_{a}$, and treats $S^{\prime K}=S^{K}+X_{a}^{K}$ as a known interference. The auxiliary random variable of the HDA Costa encoder is

$$
U^{K}=X_{h}^{K}+\alpha_{h} S^{\prime K}+\kappa_{h} V^{K}
$$

where $X_{h}$ is Gaussian $\left(\mathcal{N}\left(0, P_{h}\right)\right), \alpha_{h}=\frac{P_{h}}{P_{h}+\sigma_{W}^{2}}$, and $\kappa_{h}^{2}=$ $\frac{P_{h}^{2}}{\left(P_{h}+\sigma_{W}^{2}\right) D_{\mathrm{SI}}}$, with $D_{\mathrm{SI}}$ defined later. The HDA Costa encoder forms a codebook $\mathcal{U}$ with codeword length $K$ and $2^{K R_{h}}$ codewords ( $R_{h}$ is defined later). Every codeword is generated following the random variable $U^{K}$. The codebook is revealed to both the encoder and decoder. The encoder searches for a $U^{K} \in \mathcal{U}$ such that $\left(V^{K}, S^{\prime K}, U^{K}\right)$ are jointly typical.

At the receiver side, as shown in Fig. 4, from the first $K$ components of the received noisy sequence $Y^{N}=$ $\left[Y_{1}^{K}, Y_{2}^{N-K}\right]=X^{N}+S^{N}+W^{N}$ we get a linear MMSE estimate $V_{a}^{\prime K}$ that is used as a side information by the Wyner$\mathrm{Ziv}$ decoder to get a better estimate $V^{\prime K}$. This requires

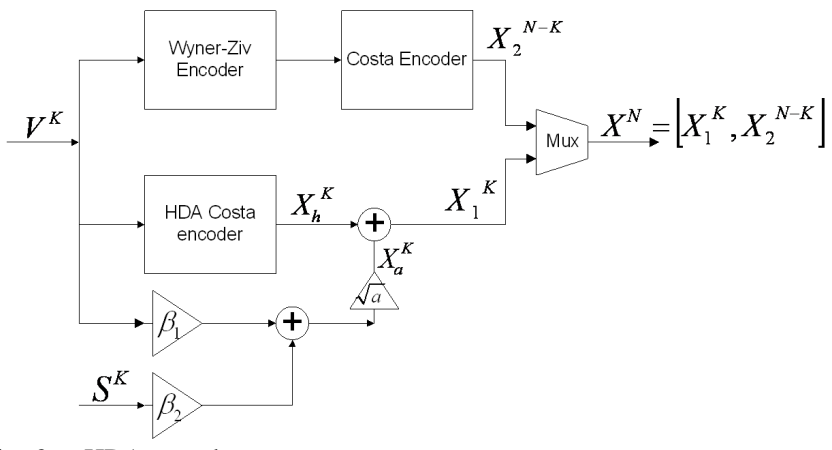

Fig. 3. HDA encoder structure.

that $R\left(D_{\text {Upper }}\right)=(r-1) C(P)$, where the rate distortion function of a Gaussian source is $R\left(D_{\text {Upper }}\right)=\frac{1}{2} \log \left(\frac{D^{*}}{D_{\text {Upper }}}\right)$, $D^{*}=\mathbb{E}\left[\left\|V^{K}-V_{a}^{\prime K}\right\|^{2}\right] / K$, and $C(P)=\frac{1}{2} \log \left(1+\frac{P}{\sigma_{W}^{2}}\right)$ is the capacity of the AWGN channel. Therefore, the distortion in $V^{\prime K}$ is

$$
D_{\text {Upper }}=D^{*}\left(1+\frac{P}{\sigma_{W}^{2}}\right)^{(1-r)}
$$

where $D^{*}$ is given by

$$
D^{*}=\sigma_{V}^{2}-\frac{\left(\sqrt{a} \beta_{1}^{2} \sigma_{V}^{2}+\left(\sqrt{a} \beta_{2}+1\right) \rho \sigma_{V} \sigma_{S}\right)^{2}}{P+\sigma_{W}^{2}+\left(2 \sqrt{a} \beta_{2}+1\right) \sigma_{S}^{2}+2 \sqrt{a} \beta_{1} \rho \sigma_{V} \sigma_{S}} \text {. }
$$

The HDA Costa decoder then searches for a $U^{K}$ that is jointly typical with the received sequence $Y_{1}^{K}$ and the side information $V^{\prime K}$. After decoding $U^{K}$, an estimate $\hat{V}^{K}$ from $Y_{1}^{K}, U^{K}$, and the Wyner-Ziv codeword $T^{K}$ is obtained. This is done using a symbol-by-symbol linear MMSE estimator. Hence the achievable distortion can be written as follows

$$
D=\inf _{P_{a}, \beta_{1}, \beta_{2}}\left(\sigma_{V}^{2}-\Gamma^{T} \Lambda^{-1} \Gamma\right)
$$

where $\Lambda$ is the covariance matrix of $\left[\begin{array}{lll}U & Y_{1} & T\end{array}\right]$, and $\Gamma$ is the correlation vector between $V$ and $\left[\begin{array}{lll}U & Y_{1} & T\end{array}\right]$. Note that $T^{K}$ is given by $T^{K}=\alpha_{w z} V^{K}+B^{K}$, with $\alpha_{w z}=\sqrt{1-\frac{D_{\text {Upper }}}{D^{*}}}$ and $B \sim \mathcal{N}\left(0, D_{\text {Upper }}\right)$. In the above distortion analysis, we assume that the encoding failure and decoding error probability of the HDA Costa codeword $U^{K}$ are arbitrarily small. This requires that $I\left(U ; S^{\prime}, V\right)<R_{h}<I\left(U ; Y_{1}, V^{\prime}\right)$; this condition is satisfied by choosing $D_{\mathrm{SI}}$ in $\kappa_{h}$ to be $D_{\mathrm{SI}}=\frac{\sigma_{V}^{2}}{D^{*}}\left(\sigma_{V}^{2}-1\right)\left(1-\frac{D_{\mathrm{Upper}}}{D^{*}}\right)+D_{\mathrm{Upper}}$.

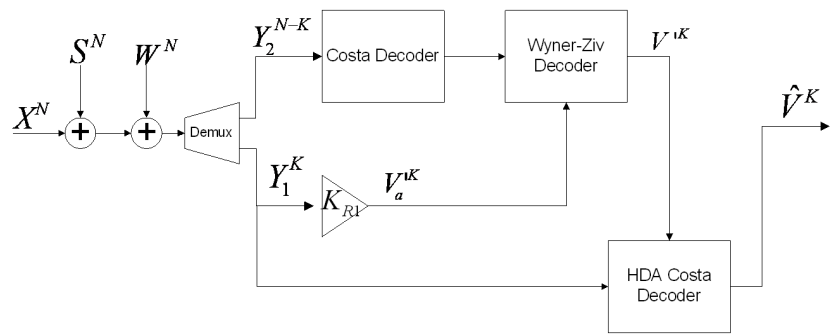

Fig. 4. HDA receiver structure. Note that $K_{R 1}$ denotes the linear MMSE estimator.

Next, we study the distortion of the proposed scheme in the presence of noise mismatch between the transmitter and the receiver. The actual channel noise power $\sigma_{W_{a}}^{2}$ is assumed to be lower than the design one $\sigma_{W}^{2}$ (i.e., $\sigma_{W_{a}}^{2}<\sigma_{W}^{2}$ ). Under such assumption, HDA Costa and Wyner-Ziv decoders are still able to find, with low probability of error, the codewords $U^{K}$ and $T^{K}$, respectively. After decoding $U^{K}, T^{K}$, a linear MMSE 
estimator of $V^{K}$ based on $Y_{1}^{K}, U^{K}$, and $T^{K}$ is calculated. Hence the achievable distortion under noise mismatch is $D_{\text {mis }}=\sigma_{V}^{2}-\Gamma^{T} \Lambda^{-1} \Gamma$, where $\sigma_{W}^{2}$ is replaced by $\sigma_{W_{a}}^{2}$ in the covariance matrix $\Lambda$. Note that all parameters $\left(P_{a}, \beta_{1}, \beta_{2}\right)$ are found from (19).

\section{B. Numerical Results}

Fig. 5 shows the performance of the proposed HDA scheme for 1:2 bandwidth expansion under matched noise level. We can notice that the proposed scheme performs close to our best derived SDR upper bound (based on (4)) and outperforms the tandem digital Costa scheme. In contrast to the digital Costa scheme, the performance of the HDA scheme increases with the correlation coefficient $\rho$. Moreover, the scheme is shown to achieve the theoretical limit for $\rho=0$ and 1. Next, we

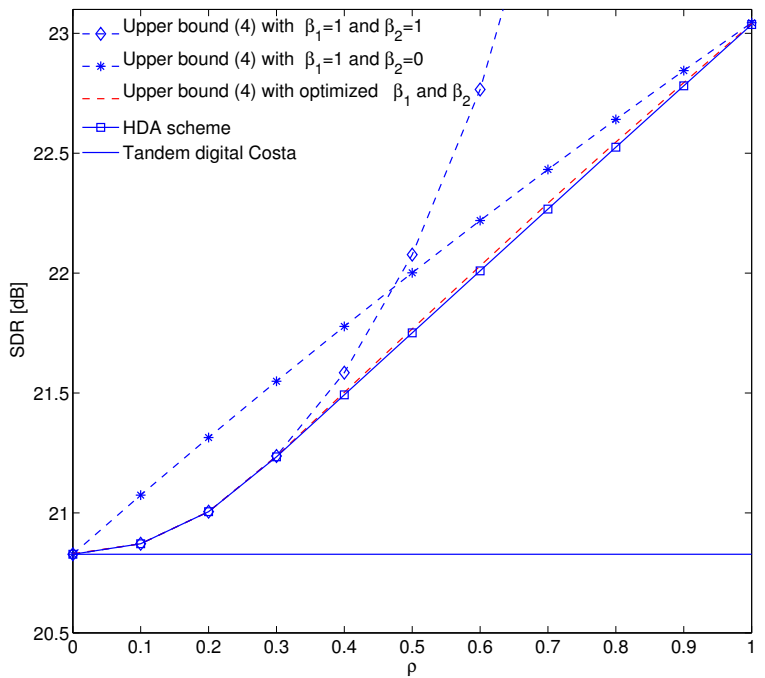

Fig. 5. Performance of 1:2 bandwidth expansion under matched noise level Digital Costa scheme and two upper bounds on SDR are plotted. The graph is made for $P=10, \sigma_{S}^{2}=1$ and $\mathrm{CSNR}=10 \mathrm{~dB}$.

design the system for $\mathrm{CSNR}=10 \mathrm{~dB}$, while the actual CSNR varies between $10 \mathrm{~dB}$ and $30 \mathrm{~dB}$. As shown in Fig. 6, we can notice that the proposed scheme is shown to be resilient under noise mismatch.

\section{SUMmARY AND CONCLUSIONS}

In this paper, we considered low/high-delay schemes for reliably transmitting Gaussian sources over AWGN channels with correlated interference under bandwidth expansion. For the low-delay regime, a non-parametric approach based on a joint optimization of the encoder and decoder was presented. Numerical results have shown that the non-parametric approach outperforms the linear scheme. The performance of a high-delay HDA scheme was analyzed under matched and mismatched noise levels between transmitter and receiver. Moreover, a lower bound on the system's distortion was derived for bandwidth expansion and the used correlation model. Future work includes the analysis of our correlated interference system under bandwidth compression.

\section{REFERENCES}

[1] M. Costa, "Writing on dirty paper," IEEE Trans. Inform. Theory, vol. 29, no. 3, pp. 439-441, May 1983.

[2] C. E. Shannon, "Communication in the presence of noise," in Proc. IRE, 1949, pp. 10-21.

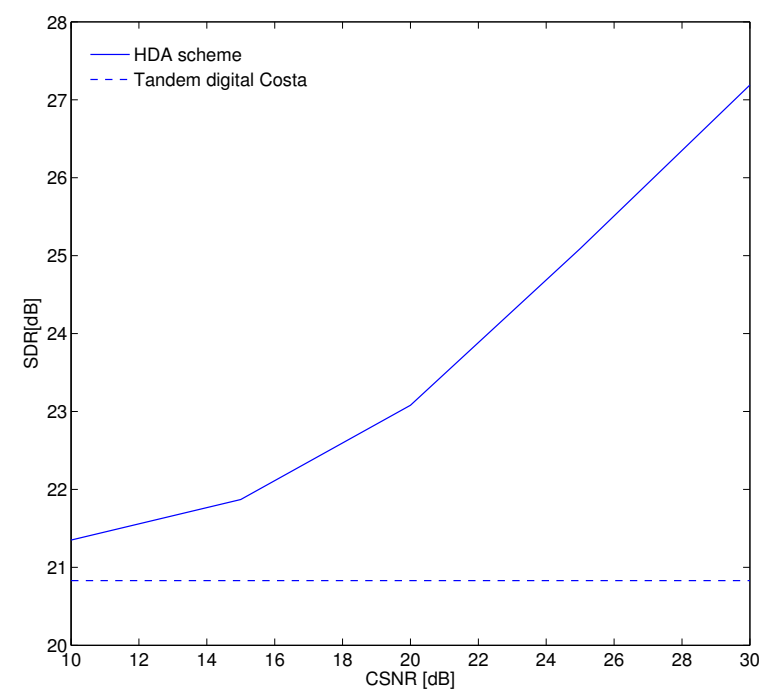

Fig. 6. Performance of 1:2 bandwidth expansion under mismatched noise level. The system is designed for $\mathrm{CSNR}=10 \mathrm{~dB}$ and $\rho=0.35$ while the actual CSNR varies from 10 to $30 \mathrm{~dB}$. Note that $P=10$ and $\sigma_{S}^{2}=1$.

[3] U. Mittal and N. Phamdo, "Hybrid digital analog (HDA) joint source channel codes for broadcasting and robust communications," IEEE Trans. Inform. Theory, vol. 48, pp. 1082-1102, May 2002.

[4] M. Skoglund, N. Phamdo, and F. Alajaji, "Hybrid digital-analog sourcechannel coding for bandwidth compression/expansion," IEEE Trans. Inform. Theory, vol. 52, no. 8, pp. 3757-3763, Aug 2006.

[5] M. P. Wilson, K. R. Narayanan, and G. Caire, "Joint source-channel coding with side information using hybrid digital analog codes," IEEE Trans. Inform. Theory, vol. 56, no. 10, pp. 4922-4940, Oct 2010.

[6] H. Behroozi, F. Alajaji, and T. Linder, "On the performance of hybrid digital-analog coding for broadcasting correlated Gaussian sources," IEEE Trans. Commun., vol. 59, no. 12, pp. 3335-3342, Dec 2011.

[7] M. Varasteh and H. Behroozi, "Optimal HDA schemes for transmission of a Gaussian source over a Gaussian channel with bandwidth compression in the presence of an interference," IEEE Trans. Signal Processing, vol. 60, no. 4, pp. 2081-2085, April 2012.

[8] Y.-C. Huang and K. R. Narayanan, "Joint source-channel coding with correlated interference," IEEE Trans. Commun., vol. 60, no. 5, pp. 13151327, May 2012.

[9] T. A. Ramstad, "Shannon mappings for robust communication," Telektronikk, vol. 98, no. 1, pp. 114-128, 2002.

[10] F. Hekland, P. A. Floor, and T. A. Ramstad, "Shannon-Kotel'nikov mappings in joint source-channel coding," IEEE Trans. Commun., vol. 57, no. 1, pp. 94-105, Jan 2009.

[11] P. A. Floor, On the Theory of Shannon-Kotel'nikov Mappings in Joint Source-Channel Coding. Ph.D. dissertation, Norwegian University of Science and Technology, 2008.

[12] Y. Hu, J. Garcia-Frias, and M. Lamarca, "Analog joint source channel coding using non-linear mappings and MMSE decoding," IEEE Trans. Commun., vol. 59, no. 11, pp. 3016-3026, Nov 2011.

[13] E. Akyol, K. Rose, and T. Ramstad, "Optimal mappings for joint source channel coding," in IEEE Inform. Theory Works., Cairo, Egypt, 2010.

[14] A. Abou Saleh, F. Alajaji, and W.-Y. Chan, "Power-constrained bandwidth-reduction source-channel mappings for fading channels," in Proc. of the 26th Bien. Symp. on Comm., Kingston, ON, May 2012.

[15] M. Skoglund and E. G. Larsson, "Optimal modulation for known interference," IEEE Trans. commun., vol. 56, no. 11, pp. 1892-1899, Nov 2008.

[16] Y.-K. Chia, R. Soundararajan, and T. Weissman, "Estimation with a helper who knows the interference," in Proc. IEEE Int. Symp. on Inform. Theory, Cambridge, MA, July 2012.

[17] V. Vaishampayan and N. Farvardin, "Joint design of block source codes and modulation signal sets," IEEE Trans. Information Theory, vol. 38, pp. 1230-1249, July 1992.

[18] J. (Karlsson) Kron and M. Skoglund, "Optimized low-delay sourcechannel-relay mappings," IEEE Trans. Commun., vol. 58, no. 5, pp. 1397-1404, May 2010. 